\title{
Mifepristone and Protection of Purkinje Neurons
}

\author{
Abdel Moumen Ghoumari* \\ Inserm University Paris Sud, Kremlin Bicêtre, France
}

Submission: July 07, 2017; Published: July 31, 2017

*Corresponding author: Abdel Moumen Ghoumari, UMR1195 Inserm University Paris Sud, 94276, Kremlin-Bicêtre, France, Email: abdel.ghoumari@inserm.fr

\section{Wide spectrum of Mifepristone use}

Mifepristone (RU486) is a synthetic steroid. By binding to the corresponding receptors, it is opposed to the activity of progesterone and gluco corticosteroids [1]. The discovery of the RU486 in the early 1980s made it possible to be used in the early voluntary terminations of pregnancy. Since this date, this molecule has not ceased to raise controversies because of its use for the abortion. Although the RU486 is widely used in USA, Canada, Europe, China, India, South Africa, etc, clinical research has not being developed without difficulty of marketing. The FDA, in September 2000, legalized its use in the United States, based on its efficacy and very good tolerance. The use of mifepristone for early abortion has become more common in the United States during the past 15 years, based on cumulative evidence indicating it safety and effectiveness [2,3].

Other than its application in the abortion, Mifepristone is also used in many fields of research such as the treatment of meningiomas, uterine fibroids, certain breast cancers, and because of its anticortisol action in the treatment of Cushing syndromes [4], and in the treatment of major psychotic depressions [5], endometriosis [6]. The neuroprotective role of RU486 was also suggested due to its anti-excitotoxic effect especially for the treatment of cranial trauma. Earlier studies have shown that RU486 can preserve hippocampal neurons treated in primary or organotypic culture, agents such as glutamate, $\beta$-amyloid or hydrogen peroxide [7]. McCullers et al. [8] also observed a neuroprotective effect of RU486 on rat hippocampal neurons after cranial trauma.

\section{Mifepristone protects Purkinje cells from death by apoptosis}

In our team, we have used mifepristone to counteract the effect of the steroid progesterone on myelination [9], using the organotypic culture model of mice or rat cerebellum. This consists in depositing cerebellar slices with a thickness of $\sim 350 \mu \mathrm{m}$ on a permeable membrane placed on a nutrient medium [9-11]. The cells thus have access to both the nutrient medium and the oxygen and the organization of the tissue remains almost identical to that observed in vivo. We have previously demonstrated that in organotypic cultures of rat or mice cerebellum, Purkinje cell death by apoptosis is age dependent [11]. Most of these cells degenerate when cerebellar slices are taken between postnatal day 1 (P1) and P5, but they survive before or after this period. This critical period of Purkinje cell vulnerability corresponds to a time window when Purkinje cells are engaged in intense synaptogenesis, dendritic remodeling, and cell death [12-14]. Surprisingly, when we did these experiments on organotypic cultures of cerebellar slices taken from rats and mice during the first postnatal week, we observed an additional effect of the anti-steroid, mifepristone. At P3, RU486 at doses $\geq 5 \mu \mathrm{M}$ prevents the apoptotic death of Purkinje cells. The same result is observed throughout the period P1-P5 of ineluctable death of the Purkinje neurons (Figure 1).
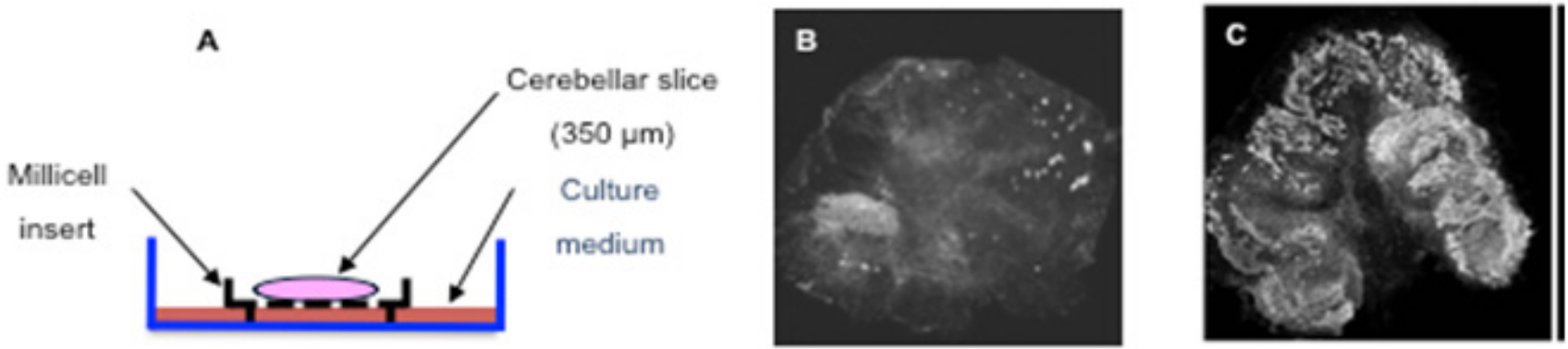

Figure 1: Mifepristone protects Purkinje cells from death in cerebellar slice culture.A: organotypic slice culture model; B-C: slice taken from cerebellum of postnatal day 3 mice and cultured for 5 days without mifepristone treatment (B) or mifepristone treated slice (C). Purkinje cells are labeled with calbindin (white color). Magnificationx5. 


\section{Open Access Journal of Neurology \& Neurosurgery}

\section{Mechanism of action of the Mifepristone (RU486)}

The mechanism of action of the RU486 in the cerebellum remains unknown. Since the anti-steroid acts conventionally at the progesterone receptor (PR) or the glucocorticosteroid receptor (GR), we investigated its effect on cerebellum explants from animals in which either of these nuclear receptors had been inactivated (PR-KO and GR-KO). Importantly, we also observed that neither progesterone nor glucocorticosteroid had any effect on the viability of Purkinje cells in organotypic cultures. The PR receptor does not appear to be involved because RU486 remained effective on cerebellar slices from PR-KO mice (in which PR was inactivated). The neuroprotective effect of RU486 is probably not related to an antioxidant effect, as suggested by [7], since antioxidants such as NAC (Nacetylcystein), vitamin E, Glutathione and MnTBAP, alone or in combination, do not prevent the apoptotic death of Purkinje cells [15].

Microarray analysis revealed that mifepristone downregulated mRNA levels of the $\mathrm{Na}+\mathrm{K}+\mathrm{ATPase}$ alpha3 subunit more than three times. Consistent with the down regulation of this protein, mifepristone caused Purkinje cell membrane depolarization. These results suggest a role of excitatory inputs in Purkinje cell survival during early postnatal development. Indeed, co-culturing cerebellar slices with glutamatergic inferior olivary neuron preparations allowed rescue of Purkinje cells. These findings reveal a new neuro protective mechanism of mifepristone and support a pivotal role for excitatory inputs in the survival of Purkinje neurons [16]. Thus, a certain degree of excitation may be necessary for Purkinje cells to survive during the early postnatal period, corresponding to a developmental stage when intense cerebellar remodeling and synaptogenesis take place. So, does neurotransmitter-induced depolarization sustain this neuro protection? During the first postnatal week, the neurotransmitter gama-aminobutyric acid (GABA) undergoes a developmental switch from being predominantly depolarizing-excitatory for immature neurons to become predominantly hyperpolarizinginhibitory for adult neurons [17]. Thus, GABA may also play a role in the viability of Purkinje neurons.

In P3 mouse cerebellar slice cultures, we show that GABAA receptor activation is depolarizing and excitatory. Antagonists of GABAA receptors rescue Purkinje neurons, demonstrating that GABA is endogenously released in this preparation and is toxic. Moreover, the p38 mitogen-activated protein kinase (MAPK) inhibitor SB239063 also, rescues Purkinje neurons [18]. In summary, we propose that when GABA is depolarizing, mifepristone protects Purkinje neurons by shunting GABA responses and probably chloride fluxes, by inhibiting p38 MAPK activity and likely internal calcium store release. Our results suggest that mifepristone may represent a useful tool in clinical practice to prevent neuro degeneration in developing brain, when exposed to several insults such as ischemia, epilepsy. It is therefore crucial to evaluate the effect of mifepristone on other types of neurons, in different brain regions, particularly in certain models of neurodegenerative diseases.

\section{References}

1. Baulieu EE (1989) Contragestion and other clinical applications of RU 486 , an antiprogesterone at the receptor. Science 245(4924): 13511357.

2. Greene MF, Drazen JM (2016) A New Label for Mifepristone. N Engl J Med 374(23): 2281-2282.

3. Simmonds KE, Beal MW, Eagen-Torkko MK (2017) Updates to the US Food and Drug Administration Regulations for Mifepristone: Implications for Clinical Practice andAccess to Abortion. J Midwifery Womens Health 62(3): 348-352.

4. Morgan FH, Laufgraben MJ (2013) Mifepristone for management of Cushing's syndrome. Pharmacotherapy 33(3): 319-329.

5. Blasey CM, Block TS, Belanoff JK, Roe RL (2011) Efficacy and safety of mifepristone for the treatment of psychotic depression. J Clin Psychopharmacol 31(4): 436-440.

6. Zhang YX (2016) Effect of mifepristone in the different treatments of endometriosis. Clin Exp Obstet Gynecol 43(3): 350-353.

7. Behl C, Trapp T, Skutella T, Holsboer F (1997) Protection against oxidative stress induced neuronal cell death-a novel role for RU486. Eur J Neurosci 9:912-20.

8. McCullers DL, Sullivan PG, Scheff SW, Herman JP (2002) Mifepristone protects CA1 hippocampal neurons following traumatic brain injury in rat. Neuroscience 109(2): 219-230.

9. Ghoumari AM, Ibanez C, El-Etr M, Leclerc P, Eychenne B, et al. (2003) Progesterone and its metabolites increase myelin basic protein expression in organotypic slice cultures of rat cerebellum. J Neurochem 86(4): 848-859.

10. Stoppini L, Buchs PA, Muller D (1991) A simple method for organotypic cultures of nervous tissue. J Neurosci Methods 37(2): 173-182.

11. Ghoumari AM, Wehrlé R, Bernard O, Sotelo C, Dusart I (2000) Implication of Bcl-2 and Caspase-3 in age-related Purkinje cell death in murine organotypic culture: an in vitro model to study apoptosis. Eur J Neurosci 12(8): 2935-2949.

12. Dusart I, Airaksinen MS, Sotelo C (1997) Purkinje cell survival and axonal regeneration are age dependent: an in vitro study. J Neurosci 17(10): 3710-3726.

13. Marin Teva JL, Dusart I, Colin C, Gervais A, van Rooijen N, et al. (2004) Microglia promote the death of developing Purkinje cells. Neuron 41(4): 535-547.

14. Armengol JA, Sotelo C (1991) Early dendritic development of Purkinje cells in the rat cerebellum. A light and electron microscopic study using axonal tracing in 'in vitro' slices. Brain Res Dev Brain Res 64(1-2): 95114

15. Ghoumari AM, Dusart I, El-Etr M, Tronche F, Sotelo C, et al. (2003) Mifepristone (RU486) protects Purkinje cells from cell death in organotypic slice cultures of postnatal rat and mouse cerebellum. Proc Natl Acad Sci USA 100(13): 7953-7958.

16. Ghoumari AM, Piochon C, Tomkiewicz C, Eychenne B, Levenes C, et al. (2006) Neuroprotective effect of mifepristone involves neuron depolarization. FASEB J 20(9): 1377-1386.

17. Ben Ari Y (2002) Excitatory actions of gaba during development: the nature of the nurture. Nat Rev Neurosci 3(9): 728-739.

18. Rakotomamonjy J, Levenes C, Baulieu EE, Schumacher M, Ghoumari AM (2011) Novel protective effect of mifepristone on detrimental GABAA receptor activity to immature Purkinje neurons. FASEB J 25(11): 3999-4010. 
